Supporting Information for

\title{
Confinement-Dependent Diffusiophoretic Transport of Nanoparticles in Collagen Hydrogels
}

\author{
Viet Sang Doan ${ }^{1}$, SungGyu Chun ${ }^{2}$, Jie Feng $^{2}, 3$, and Sangwoo Shin ${ }^{1 *}$ \\ ${ }^{1}$ Department of Mechanical and Aerospace Engineering, University at Buffalo, The State University of New \\ York, Buffalo, NY 14260, USA \\ ${ }^{2}$ Department of Mechanical Science and Engineering, University of Illinois at Urbana-Champaign, Urbana, \\ IL 61801, USA \\ ${ }^{3}$ Materials Research Laboratory, University of Illinois at Urbana-Champaign, Urbana, IL 61801, USA \\ * Correspondence: sangwoos@buffalo.edu
}

\section{Experimental Details}

\subsection{Materials}

Collagen type I solutions were purchased from Advanced BioMatrix (San Diego, CA). Polydimethylsiloxane (PDMS) was purchased from Dow Chemical (Midland, MI). Amine-modified polystyrene (aPS) particles with radius $a=100 \mathrm{~nm}$ were purchased from ThermoFisher (Waltham, MA). aPS particles with $a=50 \mathrm{~nm}$ and $500 \mathrm{~nm}, 10 \times$ phosphate-buffered saline (PBS), potassium acetate $(\mathrm{KAc})$, sodium chloride $(\mathrm{NaCl})$, sodium hydroxide $(\mathrm{NaOH})$, tetrahydrofuran $(\mathrm{THF})$, glucose and 8-hydroxypyrene-1,3,6-trisulfonic acid (HPTS) were purchased from Sigma-Aldrich (St. Louis, MO). Polystyrene (PS, $1.9 \mathrm{kDa}$ ) and polystyrene- $b$-poly(ethylene glycol) (PS- $b$-PEG, 1.6 $\mathrm{kDa}-b-5 \mathrm{kDa}$ ) were purchased from Polymer Source Inc. (Montreal, Quebec, Canada). Hostasol Yellow 3G (HY3G) was purchased from Clariant (Charlotte, NC).

\subsection{Synthesis and characterization of PS-PEG NPs}

PS-PEG NPs were fabricated via Flash NanoPrecipitation (FNP) using a confined impinging jet (CIJ) mixer. The core materials (PS and HY3G) and block copolymer (PS- $b$-PEG) were dissolved in THF at the desired concentrations. The concentration in THF was varied from 2.5 to 17.5 $\mathrm{mg} / \mathrm{mL}$ for PS and 2.5 to $7.5 \mathrm{mg} / \mathrm{mL}$ for PS- $b$-PEG, while HY3G content was kept as $1 \mathrm{wt} \%$ of the total formulation mass. In the CIJ mixer, an organic stream of THF with dissolved PS, HY3G, and PS- $b$-PEG was rapidly mixed against a deionized water (DI) water stream into the mixing chamber in a 1:1 volume ratio. The exit stream of the mixer was collected in a quenching DI water bath such that the final solvent composition was $10 \mathrm{vol} \%$ THF and $90 \mathrm{vol} \%$ DI water. Then, the remaining THF was removed from the solution by a rotary evaporator (RV 10, IKA, NC), operating at temperature $40^{\circ} \mathrm{C}$ and pressure 357 mbar for 1 hour. After the process, the samples were subject to the dynamic light scattering (DLS) characterization.

NPs samples were characterized by DLS to measure the average diameter and polydispersity index (PDI), using Litesizer 500 (Anton Paar, Graz, Austria). All measurements were performed in triplicate at $25^{\circ} \mathrm{C}$ using a detection angle of $175^{\circ}$. Mean values were reported with standard deviation. DLS data were processed with Anton Paar's software by using intensity-weighted size distributions of NPs from a distribution analysis. Zeta potential was also measured by DLS and calibrated using the Henry function [1]. 


\subsection{Collagen-embedded microfluidic setup}

The PDMS channel was made using the conventional soft lithography method. Uncured collagen solution was first mixed with $10 \times \mathrm{PBS}$. Then, $0.5 \mathrm{M} \mathrm{NaOH}$ solution was used to adjust the solution to $\mathrm{pH}=7$. Subsequently, DI water was added to the mixture to maintain the amount of $10 \times$ PBS as $1 / 10$ of the total volume. The mixing steps were performed on ice to prevent collagen from gelation. Collagen was then immediately filled inside the dead-end pores using a syringe. An empty syringe was used to flush out the collagen in the main channel, leaving only the microfluidic dead-end pores filled with collagen. The channels were then placed inside a humid incubator at $37^{\circ} \mathrm{C}$ for 90 minutes for gel formation.

\subsection{Diffusiophoresis and diffusion measurements}

After gelation, the channel was flushed with inner solution (concentration, $c_{i}$ ) for 30 minutes. Outer solution $\left(c_{o}\right)$ with NPs then was infused to induce diffusiophoresis. The fluorescent NPs were visualized by a fluorescence microscope (DMi8, Leica). Particle trajectories were tracked using Image J and the data was post-processed by MATLAB. The particle diffusiophoretic mobility was obtained by comparing the experimental particle trajectories to the theoretical particle front, which was obtained by integrating the particle $1 \mathrm{D}$ diffusiophoretic velocity:

$$
x(t)=\int \mathcal{M}_{d} \frac{\partial_{x} c}{c} d t
$$

where $\mathcal{M}_{d}$ is the particle diffusiophoretic mobility and $c(x, t)$ is the solute concentration profile inside a $1 \mathrm{D}$ dead-end pore given as:

$$
c(x, t)=c_{o}+\left(c_{i}-c_{o}\right) \sum_{k=0}^{\infty} b_{k} \sin \left(\frac{\lambda_{k} x}{L}\right) e^{-\frac{\lambda_{k}^{2} D_{s} t}{L^{2}}},
$$

where $b_{k}=2\left(1-\cos \lambda_{k}\right) / \lambda_{k}, \lambda_{k}=(2 k+1) \pi / 2$, and $D_{s}$ is the solute diffusivity. Particle mobility was extracted by fitting equation 1 to the particle front trajectory (figure $1 \mathrm{c}$ in the main text).

The diffusivity of the particles was obtained by either single particle tracking or collective particle diffusion experiment. Collective measurement was used to find diffusion coefficients of small particles such as HPTS dye, PS-PEG NPs $a=36$ to $98 \mathrm{~nm}$ and aPS NPs $a=50 \mathrm{~nm}$. These experiments were performed in long, slender dead-end pores. The solute concentrations inside and outside the dead-end pores were maintained equally to suppress diffusiophoresis ( $\mathrm{NaCl}$ for HTPS dye and PS-PEG NPs; KAc for aPS NPs). Particles were suspended in the outer flow, which were then gradually diffused into the dead-end pores (figure S1). By analyzing the fluorescence intensity across the pores, the normalized NP concentration along the pores was plotted using ImageJ and MATLAB (figure S1b). Due to the geometry in which the particles diffuse through being a long and narrow dead-end pore (length $=800 \mu \mathrm{m}$, width $=$ height $=40 \mu \mathrm{m}$ ), and due to the relatively slow

diffusion of NPs, the diffusion of the NPs inside the pore can be approximated as a 1D semi-infinite diffusion, where the NP concentration $n_{p}(x, t)$ is given as:

$$
n_{p}(x, t)=n_{p 0}\left[1-\operatorname{erf}\left(\frac{x}{\sqrt{4 D_{p} t}}\right)\right],
$$

where $n_{p 0}$ is the particle concentration at the pore entrance and $D_{p}$ is the particle diffusivity. The diffusivity was determined by adjusting $D_{p}$ to fit equation 3 to the measured data (figure S1b).

The diffusivity of large aPS NPs $(a=100 \mathrm{~nm})$ was measured by tracking the random-walk trajectories using ImageJ. The plot of mean squared displacement (MSD) and the lag time between 

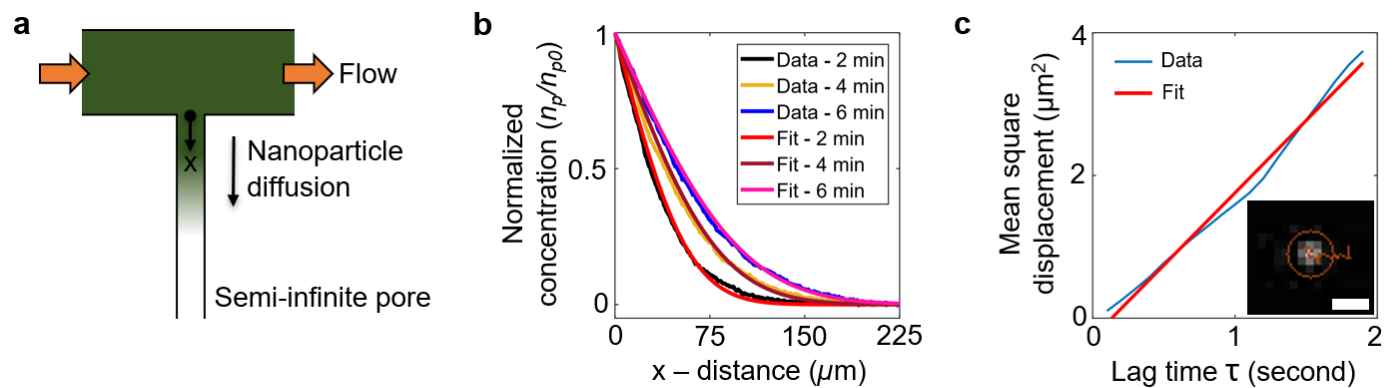

Figure S1. Particle diffusivity measurements. (a) 1D diffusion setup in semi-infinite pore where background solute concentration was the same inside and outside the pore to suppress diffusiophoresis and only allow diffusion. (b) Normalized particle concentration $n_{p} / n_{p 0}$ along the pore with different diffusion time of $a=$ $36 \mathrm{~nm}$ PS-PEG NPs in free space. (c) Mean squared displacement of aPS NPs $(a=100 \mathrm{~nm} \mathrm{in} 1.8 \mathrm{mg} / \mathrm{ml}$ collagen) versus the lag time between two particle locations $\tau$. Inset is the particle trajectory obtained by ImageJ. The scale bar is $2 \mu \mathrm{m}$.

two particle locations $\tau$ was generated by MATLAB. A linear line (red line) was created to find the slope $\alpha$ (figure $\mathrm{S} 1 \mathrm{c}$ ). The diffusion coefficient is then calculated as $D=\alpha / 4$.

\section{Theory}

\subsection{Electrophoresis of a spherical particle in a spherical cavity}

Based on the Zydney's model for electrophoresis of a charged particle confined in a spherical cavity at arbitrary $\lambda_{1}$ and $\lambda_{2}$, where $\lambda_{1}=\kappa a$ and $\lambda_{2}=a / h$, the size-dependent electrophoretic mobility $\mathcal{M}_{e}$ reads $[2]$

$$
\mathcal{M}_{e}\left(\lambda_{1}, \lambda_{2}\right)=\frac{\epsilon \zeta}{\mu}\left[\mathcal{A}-\mathcal{B}\left(\frac{1-\lambda_{2}^{2}}{1-\lambda_{2}^{5}}\right)\right]
$$

where $\epsilon$ is the fluid permittivity, $\zeta$ is the particle zeta potential, and $\mu$ is the fluid viscosity. $\mathcal{A}$ and $\mathcal{B}$ are both functions of $\lambda_{1}$ and $\lambda_{2}$, given as

$$
\begin{aligned}
& \mathcal{A}=\frac{1}{1-\lambda_{2}^{2}}\left[1+2 \int_{\lambda_{2}}^{1}\left(\frac{\lambda_{2}^{3}}{\eta^{4}}-\frac{5 \lambda_{2}^{5}}{\eta^{6}}\right) \xi \mathrm{d} \eta\right], \\
& \mathcal{B}=\frac{5}{1-\lambda_{2}^{2}}\left[\frac{\lambda_{2}^{3}}{2}+\int_{\lambda_{2}}^{1}\left(\eta^{2}-\frac{\lambda_{2}^{8}}{\eta^{6}}\right) \xi \mathrm{d} \eta\right],
\end{aligned}
$$

where $\eta=r / h . \xi$ is given as

$$
\xi(\eta)=\frac{\lambda_{2}}{\eta}\left[\cosh \left(\frac{\lambda_{1}}{\lambda_{2}} \eta-\lambda_{1}\right)-\operatorname{coth}\left(\frac{\lambda_{1}}{\lambda_{2}}-\lambda_{1}\right) \cdot \sinh \left(\frac{\lambda_{1}}{\lambda_{2}} \eta-\lambda_{1}\right)\right] .
$$

In the limit of thin Debye layer $\left(\lambda_{1} \rightarrow \infty\right)$, equation 4 reduces to the following exact solution as

$$
\mathcal{M}_{e}\left(\lambda_{2}\right)=\frac{\epsilon \zeta}{\mu}\left[\frac{2-5 \lambda_{2}^{3}+3 \lambda_{2}^{5}}{2\left(1-\lambda_{2}^{3}\right)\left(1-\lambda_{2}^{5}\right)}\right],
$$

which is plotted as black dash-dot curves in figure 4 in the main text. 


\subsection{Diffusiophoresis of a spherical particle with a polarizable Debye layer in a spherical cavity}

Chiu and Keh recently obtained a closed-form solution for the diffusiophoresis of weakly charged particles immersed in a symmetric electrolyte inside a spherical cavity in the limit of thin $\left(\lambda_{1}>\right.$ $\mathcal{O}(10)$ ), but polarizable Debye layer [3]. The diffusiophoretic mobility $\mathcal{M}_{d}$ reads

$$
\mathcal{M}_{d}\left(\lambda_{1}, \lambda_{2}\right)=\frac{2 \epsilon}{\mu}\left(\frac{k_{B} T}{z e}\right)^{2}\left[\frac{5}{3}\left(\frac{1+\lambda_{2}+\lambda_{2}^{2}}{1+\lambda_{2}+\lambda_{2}^{2}+\lambda_{2}^{3}+\lambda_{2}^{4}}\right)-1\right] \mathcal{C},
$$

where $k_{B}$ is the Boltzmann constant, $T$ is the temperature, $z$ is the valence of the solute species, and $e$ is the elementary charge. $\mathcal{C}$ is given as

$$
\mathcal{C}=2 \beta \bar{\zeta}+2 \ln \cosh \bar{\zeta}+\left(1-\lambda_{2}^{3}\right) \cdot\left[\left(\alpha_{1}-\alpha_{2}\right) \bar{\zeta}+\left(\alpha_{1}+\alpha_{2}\right) \ln \cosh \bar{\zeta}\right],
$$

where $\beta=\left(D_{-}-D_{+}\right) /\left(D_{-}+D_{+}\right)$is the dimensionless diffusivity contrast, $D_{ \pm}$is the diffusivity of cation $(+)$ and anion $(-)$, and $\bar{\zeta}=z e \zeta / 4 k_{B} T$ is the dimensionless zeta potential. $\alpha_{1}$ and $\alpha_{2}$ are

$$
\begin{aligned}
& \alpha_{1}=\frac{3 \tau_{+}+(1+\beta)\left(\theta_{1} \delta_{1}+\delta_{3} \lambda_{2}^{3}\right)}{\delta_{1}+\delta_{2} \lambda_{2}^{3}+\delta_{3} \lambda_{2}^{6}}, \\
& \alpha_{2}=\frac{3 \tau_{-}+(1-\beta)\left(\theta_{2} \delta_{1}+\delta_{3} \lambda_{2}^{3}\right)}{\delta_{1}+\delta_{2} \lambda_{2}^{3}+\delta_{3} \lambda_{2}^{6}} .
\end{aligned}
$$

Here, $\theta_{1}, \theta_{2}, \delta_{1}, \delta_{2}, \delta_{3}, \gamma_{ \pm}, \tau_{ \pm}$, and $f_{ \pm}$, are defined as

$$
\begin{gathered}
\theta_{1}=\frac{1-2 \gamma_{+}+\gamma_{-}-2 \gamma_{+} \gamma_{-}-3 \tau_{+}+2 \tau_{+} \tau_{-}}{2 \delta_{1}}, \\
\theta_{2}=\frac{1+\gamma_{+}-2 \gamma_{-}-2 \gamma_{+} \gamma_{-}-3 \tau_{-}+2 \tau_{+} \tau_{-}}{2 \delta_{1}}, \\
\delta_{1}=1+\gamma_{+}+\gamma_{-}+\gamma_{+} \gamma_{-}-\tau_{+} \tau_{-}, \\
\delta_{2}=\delta_{1}\left(\theta_{1}+\theta_{2}\right)+\frac{3}{2}\left(\tau_{+}+\tau_{-}\right), \\
\delta_{3}=\frac{9}{4}-\left(\delta_{1}+\delta_{2}\right), \\
\gamma_{ \pm}\left[\left(1+\frac{3 f_{ \pm}}{z^{2}}\right) \exp ( \pm \bar{\zeta}) \sinh \bar{\zeta}-\frac{3 f_{ \pm}}{z^{2}}(\bar{\zeta} \pm \ln \cosh \bar{\zeta})\right] \\
\tau_{ \pm}=\frac{12 f_{ \pm}}{\lambda_{1} z^{2}} \ln \cosh \bar{\zeta} \\
f_{ \pm}=\frac{2 \epsilon}{3 \mu D_{ \pm}}\left(\frac{k_{B} T}{z e}\right)^{2} .
\end{gathered}
$$

\subsection{Hindered diffusion in fibrous media}

The hindered diffusive transport in porous media is attributed to two major factors, namely the steric- and the hydrodynamic effects. Brady has proposed a simple expression for the diffusivity hindrance as $[4,5]$

$$
\frac{D}{D_{0}}=\mathcal{F} \cdot \mathcal{S}
$$


where $\mathcal{F}$ is a factor that takes into account the hydrodynamic interaction and $\mathcal{S}$ is the steric factor that depends on the pore geometry and the particle size. $\mathcal{F}$ is given by solving the Brinkman equation as $\mathcal{F}=\left[1+a / k^{1 / 2}+a^{2} /(9 k)\right]^{-1}$, and $k$ is the Darcy permeability of the media $[4,6]$. For collagen type I hydrogels, Ramanujan et al. previously measured the Darcy permeability of collagen matrix for various collagen concentrations, where the power law expression can be obtained from the measured data as $k=30854 w^{-1.442}$, where $w$ is the gel concentration in $[\mathrm{mg} / \mathrm{ml}]$ and $k$ is the Darcy permeability in $\left[\mathrm{nm}^{2}\right][7]$.

The Darcy permeability $k$ can be used to obtain the steric factor $\mathcal{S}$ and the effective pore radius $h$. Johansson and Löfroth obtained $\mathcal{S}$ for polymeric hydrogels via Brownian dynamics simulations, given as $\mathcal{S}=\exp \left(-0.84 f^{1.09}\right)$, where $f=(1+a / t)^{2} \phi$ is the adjusted volume fraction of the polymers, $\phi$ is the volume fraction, $t$ is the polymer (fiber) radius $(\approx 162 \mathrm{~nm}$ for type I collagen [8]) [9]. $\phi$ can be determined from the Darcy permeability $k$ and the fiber thickness $t$ using the expression obtained by Jackson and James for various fibrous porous media including collagen, given as $k=-0.15 t^{2}(\ln \phi+0.931) / \phi$, which is shown to be accurate for various $\phi$ values ranging over three orders of magnitude [10].

The effective pore radius $h$ can be obtained by relating to $\phi$ and $k$ using the Kozeny-Carman equation, $h=\sqrt{k q /(1-\phi)}$, where $q$ is the Kozeny factor that depends on the pore geometry [11]. For a 3D porous media packed with randomly oriented cylindrical fibers, $q$ is given as $q=$ $\left(q_{\|}+2 q_{\perp}\right) / 3 . q_{\|}$and $q_{\perp}$ are Kozeny factors for flow parallel and perpendicular to the cylinder axis, respectively, which are defined as $[12,13]$

$$
\begin{gathered}
q_{\|}=\frac{2(1-\phi)^{3}}{\phi\left(-2 \ln \phi-3+4 \phi-\phi^{2}\right)}, \\
q_{\perp}=\frac{2(1-\phi)^{3}}{\phi\left(-2 \ln \phi-\frac{1-\phi^{2}}{1+\phi^{2}}\right)} .
\end{gathered}
$$

\section{References}

[1] B. J. Kirby. Micro- and Nanoscale Fluid Mechanics: Transport in Microfluidic Devices. Cambridge University Press, 2010.

[2] A. L. Zydney. Boundary effects on the electrophoretic motion of a charged particle in a spherical cavity. J. Colloid Interface Sci., 169(2):476-485, 1995.

[3] H. C. Chiu and H. J. Keh. Electrophoresis and diffusiophoresis of a colloidal sphere with double-layer polarization in a concentric charged cavity. Microfluid. Nanofluid., 21(3):45, 2017.

[4] R. J. Phillips, W. M. Deen, and J. F. Brady. Hindered transport of spherical macromolecules in fibrous membranes and gels. AIChE J., 35(11):1761-1769, 1989.

[5] E. M. Johnson, D. A. Berk, R. K. Jain, and W. M. Deen. Hindered diffusion in agarose gels: test of effective medium model. Biophys. J., 70(2):1017-1023, 1996.

[6] Y. E. Solomentsev and J. L. Anderson. Rotation of a sphere in brinkman fluids. Phys. Fluids, 8(4):1119-1121, 1996.

[7] S. Ramanujan, A. Pluen, T. D. McKee, E. B. Brown, Y. Boucher, and R. K. Jain. Diffusion and convection in collagen gels: implications for transport in the tumor interstitium. Biophys. J., 83(3):1650-1660, 2002. 
[8] P. Dutov, O. Antipova, S. Varma, J. P. R. O. Orgel, and J. D. Schieber. Measurement of elastic modulus of collagen type i single fiber. PLOS ONE, 11(1):e0145711, 2016.

[9] L. Johansson and J.-E. Löfroth. Diffusion and interaction in gels and solutions. 4. hard sphere brownian dynamics simulations. J. Chem. Phys., 98(9):7471-7479, 1993.

[10] G. W. Jackson and D. F. James. The permeability of fibrous porous media. Can. J. Chem. Eng., 64(3):364-374, 1986.

[11] A. Pluen, P. A. Netti, R. K. Jain, and D. A. Berk. Diffusion of macromolecules in agarose gels: comparison of linear and globular configurations. Biophys. J., 77(1):542-552, 1999.

[12] J. Happel and H. Brenner. Low Reynolds Number Hydrodynamics, volume 1. Springer Science \& Business Media, 2012.

[13] J. R. Levick. Flow through interstitium and other fibrous matrices. Q. J. Exp. Physiol., 72 (4):409-437, 1987. 